\title{
Incidence of Vaginal Colonization of Group B Streptococci among Pregnant Women Attending a Tertiary Care Hospital - Chennai, India
}

\author{
Jayashree Hariharan (D), Preethi Venkatesan (1) and Priyadarshini Shanmugam* \\ Department of Microbiology, Chettinad Hospital and Research Institute, Chettinad Academy of Research and \\ Education, Kelambakkam - 603 103, Tamil Nadu, India.
}

\begin{abstract}
Streptococcus agalactiae or Group B Streptococcus (GBS) is an important pathogen, which can cause serious illness and sometimes death especially in newborns, pregnant women and in people with compromised immune system. The major risk factor for neonatal disease is vaginal colonization of GBS during pregnancy and delivery. 1) To study the incidence of vaginal colonization of Group B Streptococcus (GBS) among pregnant women. 2) To compare the efficacy of sheep blood agar vs selective medium like HiCHROME Strep B Selective agar (HIMEDIA) in the isolation of GBS. Vaginal swabs were collected from 150 antenatal women at 35-37 weeks of gestation or at any gestational age if additional risk factors were present. The swabs were cultured on non-selective medium like Blood agar and a chromogenic medium (HiCHROME Strep B Agar, HiMedia). GBS was identified by standard microbiological techniques. Bluish, purple-colored colonies were confirmed as GBS by CAMP test and latex agglutination test. Only 3 out of 150 women (2\%) of the screened antenatal women were colonized with GBS. Incidence was quite low, compared to the literature from the West. All the $\mathbf{3}$ babies born to the three women colonized with GBS were healthy with no indication of invasive GBS disease. The chromogenic medium supported the growth of Group B Streptococcus and Enterococcus spp. only and was a useful selective medium for isolation of GBS.
\end{abstract}

Keywords: Neonatal meningitis, congenital infection, antenatal infection, female genital tract infection

*Correspondence: priyadarshini0018@gmail.com; +91 9841551891

(Received: December 11, 2021; accepted: January 11, 2022)

Citation: Hariharan J, Venkatesan P, Shanmugam P. Incidence of Vaginal Colonization of Group B Streptococci among Pregnant Women Attending a Tertiary Care Hospital - Chennai, India. J Pure Appl Microbiol. 2022;16(1):419-423. doi: 10.22207/JPAM.16.1.36

(c) The Author(s) 2022. Open Access. This article is distributed under the terms of the Creative Commons Attribution 4.0 International License which permits unrestricted use, sharing, distribution, and reproduction in any medium, provided you give appropriate credit to the original author(s) and the source, provide a link to the Creative Commons license, and indicate if changes were made. 


\section{INTRODUCTION}

Group B Streptococci (GBS) also known as Streptococcus agalactiae is a Gram-positive beta hemolytic Streptococcus which can cause serious illness and sometimes death especially in the newborn, pregnant women and in people with a compromised immune system. ${ }^{1}$ In general, GBS is a harmless commensal or a normal inhabitant of lower gastro-intestinal and genito-urinary tract. ${ }^{1,2}$ It can be a colonizer in $10-30 \%$ of pregnant women. Up to half of these women give birth to infants who are colonized with GBS and 2-3\% of these infants may develop invasive infections like pneumonia, septicemia or meningitis. ${ }^{2}$ In the mother, GBS may cause abortion, urinary infection, preterm birth, chorioamnionitis or puerperal endometritis. ${ }^{3}$ A hypothesis for occurrence of this infection might be the hormonal changes occurring during the gestational period and further microbiota imbalance thereby increasing the chances of GBS infection and complications. ${ }^{3}$

In India, studies have shown colonization rates comparable to the Western literature, however, GBS disease in the newborn is not well recognized and is often underestimated. ${ }^{4}$ The Center for Disease Control, USA (CDC) recommends routine screening of $G B S$ in pregnant women in many western countries and has not framed any mandate for other countries. ${ }^{4}$ In India, however this practice is not widespread. ${ }^{4}$ Studies have shown that transmission is high during child birth and can cause mortality and morbidity in the newborn. ${ }^{3}$ As a result, it is advised to adopt screening strategies between the $35^{\text {th }}$ and $37^{\text {th }}$ weeks of gestation. ${ }^{3}$ By doing so, at-risk mothers can be identified and treated with intrapartum antibiotics. ${ }^{4}$

\section{Aim \& Objectives}

1. To study the incidence of vaginal colonization of Group B Streptococcus (GBS) among pregnant women in Chennai, India.

2. To compare the efficacy of sheep blood agar vs. selective medium like HiCrome Strep B Selective agar (HIMEDIA).

\section{MATERIALS AND METHODS}

This descriptive study was carried out from April 2018 to October 2018 at a tertiary care private Hospital at Chennai. Institutional Human
Ethics Committee (IHEC) approval was obtained, after which sample collection was started.

The study included pregnant women at 35-37 weeks of gestation, antenatal women at any gestational age with history of preterm labor, chorioamnionitis or puerperal sepsis in previous pregnancies and antenatal women at any gestational age with history of neonatal infection of their newborn in prior pregnancies. Healthy nulligravida, primigravida with gestational age of less than 35 weeks and multigravida with good obstetric histories and gestational age less than 35 weeks were excluded from the study. If the women were found to be GBS carriers, they were informed about their status so that their consulting physician could decide on the use of intrapartum antibiotics to prevent GBS disease in the newborn. After obtaining written informed consent, patient history was obtained using a questionnaire and two high vaginal swabs were collected simultaneously and the samples were sent to the microbiology lab using Amie's transport medium. The samples were inoculated into sheep blood agar and a selective medium, HiCHROME Strep $B$ Selective agar. After overnight incubation, the organism was identified in culture by Gram staining and appropriate microbiological tests.

In culture, the Group B Streptococci produced large, grey colonies with a narrow zone of beta hemolysis on Blood agar and bluish to purple colonies on HiCHROME Strep B agar. On Gram staining, they appeared as Gram positive cocci in chains. They were catalase test negative. Such colonies were further subjected to the following biochemical tests for confirmation as Group B Streptococci.

\section{CAMP Test}

The CAMP test is used to identify the Group B Streptococci. CAMP stands for Christie, Atkins and Munch-Petersen, the scientists who devised this test to demonstrate the accentuated zone of hemolysis when GBS is inoculated perpendicular to a streak of Staphylococcus aureus. The production of a distinct "arrowhead" hemolysis constituted a positive CAMP reaction. HI-STREP Latex Agglutination Test

Latex particles in the HiStrep Latex test kit are individually sensitized with rabbit antibodies specific to one of the Streptococcal carbohydrate 
antigens of groups A, B, C, D, F or G. Streptococcal colonies from culture plates were incubated in an enzyme solution to extract the antigen. The extract/antigen preparation was tested on a reaction card against six suspensions of antibody coated latex particles each specific for one of the groups $A, B, C, D, F$, and $G$. In the presence of homologous antigen, particles in one of the suspensions will aggregate to give visible agglutination in contrast to the other suspensions that will remain non agglutinated. GBS agglutinated with B antisera alone.

\section{Antibiotic Susceptibility Testing}

The isolates were then tested for its susceptibility to various antibiotics by Kirby Bauer disc diffusion method. A lawn culture of the 0.5 McFarland broth suspension of beta hemolytic Streptococci were made on a blood agar plate. The sensitivity to the following antibiotics was tested. Penicillin (10U), Ampicillin (10 $\mu \mathrm{g})$, Cefotaxime $(30 \mu \mathrm{g})$, Vancomycin $(30 \mu \mathrm{g})$, Erythromycin (15 $\mu \mathrm{g})$, Azithromycin $(15 \mu \mathrm{g})$, Tetracycline $(30 \mu \mathrm{g})$, Ofloxacin $(5 \mu \mathrm{g})$, Clindamycin $(2 \mu \mathrm{g})$, Linezolid $(30 \mu \mathrm{g})$. The testing and result interpretation was performed according to the Clinical Laboratories and Standards Institute (CLSI) 2018 guidelines.

\section{RESULTS}

Over a period of 6 months, vaginal swabs were collected from 150 antenatal women visiting our hospital's antenatal clinic. Most of the women belonged to low socio-economic status. The

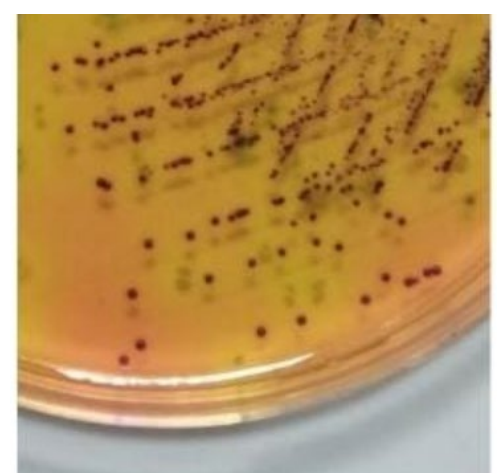

Fig. 1. The HiCHROME Strep B chromogenic media supports the growth of GBS and Enterococcus species only. GBS produced bluish purple colonies (Fig. 1) whereas Enterococcus will produce green-coloured colonies. women ranged from 19 to 35 years in age. Of the 150 high vaginal swabs subjected to bacteriological culture in Blood agar, 104 samples yielded normal vaginal flora. Group B Streptococci (GBS) was isolated from 3 women in the 23-28-year age group and all were primigravida.

Forty-six isolates were obtained in all, the details of which are given in Table 1.

The HiCHROME Strep B chromogenic media however supported the growth of GBS and Enterococcus species only. GBS produced bluish purple colonies (Fig. 1) whereas Enterococcus produced green-coloured colonies. The 3 isolates of GBS were detected both in the Blood agar and HiCHROME Strep B agar. The chromogenic media did not pick up any additional isolates of GBS.

Only three antenatal women out of 150 screened were found to harbor Group B Streptococci, indicating that $2.0 \%$ of pregnant women in our study were colonized with GBS.

These 3 women gave birth to healthy term infants with no further complications. One of these women was short statured primi with premature rupture of membranes who was taken up for an emergency LSCS. The other women had no complications during pregnancy and had normal vaginal deliveries.

\section{DISCUSSION}

The present study found a very low incidence of GBS carriage $(2.0 \%)$ in the pregnant women attending our hospital. The carriage rate of GBS in pregnant women is widely variable worldwide ranging from $1.4 \%$ to $40 \% .^{5}$ Literature from the West has shown higher rates of GBS

Table 1. Forty-six isolates were obtained in all, the details of which are given

\begin{tabular}{lc}
\hline Name & No. of Isolates \\
\hline Escherichia coli & 14 \\
Candida albicans & 11 \\
Candida non-albicans & 6 \\
Acinetobacter spp. & 3 \\
Group B Streptococcus (GBS) & 3 \\
Enterococcus spp. & 3 \\
Klebsiella spp. & 2 \\
Staphylococcus aureus & 2 \\
Citrobacter koseri & 1 \\
Enterobacter spp. & 1 \\
\hline
\end{tabular}


carriers among pregnant women. ${ }^{4}$ The prevalence rates of GBS carriage in Ethiopia, Korea, Taiwan and Tanzania were $14.6 \%, 8.3 \%, 11.6 \%$ and $23 \%$ respectively. ${ }^{6-9}$ The carriage rates found in some Indian studies has been comparable to the West, but most of the studies have found low maternal GBS carriage rates. ${ }^{10}$ In a South Indian study on GBS, the maternal carriage rate was found to be $7.5 \% .^{11}$ As invasive GBS occurs in only $1-2 \%$ of infants born to colonized mothers, very few Indian studies are available to evaluate the GBS disease in the newborn. ${ }^{4}$ Our study results revealed that most of the vaginal swabs yielded higher colonization of normal vaginal flora. This may be a predisposing factor for the lower incidence of GBS rates in the study population as higher vaginal colonization of microbiota has antagonist effect in the colonization of GBS. ${ }^{12-14}$ In our study, the three neonates were healthy and had an uneventful neonatal period. The newborns were not evaluated for their colonization status, which is a limitation in our study. As the incidence of GBS colonization in our study was found to be very low, the cost-effectiveness of universal screening of all antenatal women and incidence of invasive GBS disease in affected infants needs further looking into.

For selective screening for GBS in antenatal women, the HiCHROME Strep B media was very useful as it suppressed growth of normal flora and helped in easy identification of the GBS, which produced bluish, purple-colored the samples colonies. However, it should be kept in mind that chromogenic media support other Streptococcus species and Enterococcus species that may produce GBS-like colonies. ${ }^{15}$ Hence colonies suggestive of GBS need to be confirmed using additional biochemical tests like CAMP and Latex agglutination tests. ${ }^{15}$ On the other hand, chromogenic media, unlike Granada media, do not need anaerobic incubation and it can also detect non-hemolytic Streptococci. ${ }^{15,16}$ The advantage of using Blood agar in addition to the HiCHROME agar was that, it could detect a total of 46 bacterial and yeast isolates from the 150 vaginal swabs tested. The HiCHROME agar alone supported only Group B Streptococcus (3 isolates) and Enterococcus spp (3 isolates), both of which produced different colored colonies and hence was a good selective medium for GBS isolation. Furthermore, in our study, there was isolation of no other Streptococcus spp, which could produce colonies that mimic GBS and hence further evaluation may be necessary to determine its efficacy as selective medium for GBS alone. In our study, the incidence of Escherichia coli colonization was found to be $9.3 \%$ (14/150). Vaginal colonization of Escherichia coli is a major risk factor for neonatal meningitis, sepsis and other perinatal complications. Therefore it is necessary to screen and treat heavy vaginal colonization of E.coli in order to prevent pregnancy complications. $^{17}$

\section{CONCLUSION}

The incidence of vaginal colonization of Group B Streptococci among pregnant women attending our hospital was found to be $2 \%$. All the three neonates born to colonized women were healthy with no evidence of invasive GBS disease. As the vaginal colonization rate in our study is $2 \%$ a universal screening for GBS is not justified. However, a risk-based screening approach may be a better strategy, since our study population yielded a higher rate of vaginal E.coli colonization. A chromogenic media like HiCrome Strep B agar was useful in selective isolation GBS from colonized patients. Blood agar could pick up additional isolates and hence its inoculation is still warranted. However, due to the distinct bluish purple colored colonies produced by GBS on the HiCrome Strep B medium, GBS is less likely to be missed and is especially useful for non-hemolytic GBS strains.

\section{ACKNOWLEDGMENTS}

The authors would like to thank Dr. Anuradha CR, Professor and Head, Department of Obstetrics and Gynaecology, Chettinad Hospital and Research Institute, Chennai, India for helping us in sample collection.

\section{CONFLICT OF INTEREST}

The authors declare that there is no conflict of interest.

\section{AUTHORS' CONTRIBUTION}

$\mathrm{JH}, \mathrm{PV}$ and PS collected the samples analysed the data. JH and PV prepared the 
manuscript. JH and PS revised the manuscript. PS modified and reviewed the manuscript. All authors read and approved the final manuscript for publication.

\section{FUNDING}

This study was funded by Indian Council of Medical Research (ICMR), New Delhi, India. (Reference Id : 2018-01604).

\section{DATA AVAILABILITY}

All datasets generated or analyzed during this study are included in the manuscript.

\section{ETHICS STATEMENT}

This study was approved by the Institutional Human Ethics Committee (IHEC) (Proposal No.85/IHEC/3-18), Chettinad Academy of Research and Education, Chennai, Tamil Nadu, India.

\section{REFERENCES}

1. Alemseged G, Niguse $\mathrm{S}$, Hailekiros $\mathrm{H}$, Abdulkadir M, Saravanan M, Asmelash T. Isolation and anti-microbial susceptibility pattern of group B Streptococcus among pregnant women attending antenatal clinics in Ayder Referral Hospital and Mekelle Health Center, Mekelle, Northern Ethiopia. BMC Research Notes. 2015;8(1):518. doi: 10.1186/s13104-015-1475-3

2. Rao GG, Nartey G, McAree T, et al. Outcome of a screening programme for the prevention of neonatal invasive early-onset group B Streptococcus infection in a UK maternity unit: an observational study. BMJ Open. 2017;7(4):e014634. doi: 10.1136/ bmjopen-2016-014634

3. Melo SC, Costa AB, Silva FT, et al. Prevalence of Streptococcus agalactiae colonization in pregnant women from the $18^{\text {th }}$ Health Region of Parana State. Rev Inst Med Trop S Paulo. 2018;60. doi: 10.1590/ s1678-9946201860002

4. Narava S, Rajaram G, Ramadevi A, Prakash GV, Mackenzie S. Prevention of perinatal group $B$ streptococcal infections: A review with an Indian perspective. Indian J Med Microbiol. 2014;32(1):6-12. doi: 10.4103/0255-0857.124286

5. Bianchi-Jassir F, Seale AC, Kohli-Lynch M, et al. Preterm birth associated with group B Streptococcus maternal colonization worldwide: systematic review and metaanalyses. Clin Infect Dis. 2017;65(suppl_2):S133-S142. doi: $10.1093 / \mathrm{cid} / \mathrm{cix} 661$
6.

Assefa S, Desta K, Lema T. Group B streptococci vaginal colonization and drug susceptibility pattern among pregnant women attending in selected public antenatal care centers in Addis Ababa, Ethiopia. BMC Pregnancy and Childbirth. 2018;18(1):135. doi: 10.1186/s12884-018-1791-4

7. Matee MI, Massawe FA, Lyamuya EF, Joachim A. Maternal and neonatal colonisation of group $B$ streptococcus at Muhimbili National Hospital in Dar es Salaam, Tanzania: prevalence, risk factors and antimicrobial resistance. BMC Public Health. 2009;9(1):437. doi: 10.1186/1471-2458-9-437

8. Kim DH, Min BJ, Jung EJ, et al. Prevalence of group B streptococcus colonization in pregnant women in a tertiary care center in Korea. Obstet Gynecol Sci. 2018;61(5):575-583. doi: 10.5468/ogs.2018.61.5.575

9. Hung LC, Kung PT, Chiu TH, et al. Risk factors for neonatal early-onset group B streptococcus-related diseases after the implementation of a universal screening program in Taiwan. BMC Public Health. 2018;18(1):438. doi: 10.1186/s12889-018-5358-0

10. Schrag SJ, Zywicki S, Farley MM, et al. Group B streptococcal disease in the era of intrapartum antibiotic prophylaxis. N Eng J Med. 2000;342(1):1520. doi: 10.1056/NEJM200001063420103

11. Das A, Ray P, Sharma M, Gopalan S. Rapid diagnosis of vaginal carriage of group B beta haemolytic streptococcus by an enrichment cum antigen detection test. Indian J Med Res. 2003;117:247-252.

12. Rosen GH, Randis TM, Desai PV, et al. Group B Streptococcus and the vaginal microbiota. J Infect Dis. 2017;216(6):744-751. doi: 10.1093/infdis/jix395

13. Patras KA, Nizet V. Group B streptococcal maternal colonization and neonatal disease: molecular mechanisms and preventative approaches. Frontiers in Pediatrics. 2018;6:27. doi: 10.3389/fped.2018.00027

14. Pace RM, Chu DM, Prince AL, Ma J, Seferovic MD, Aagaard KM. Complex species and strain ecology of the vaginal microbiome from pregnancy to postpartum and association with preterm birth. Med. 2021;2(9):10271049. doi: 10.1016/j.medj.2021.06.001

15. Rosa-Fraile M, Spellerberg B. Reliable detection of group B streptococcus in the clinical laboratory. J Clin Microbiol. 2017;55(9):2590-2598. doi: 10.1128/ JCM.00582-17

16. Schuchat A. Epidemiology of group B streptococcal disease in the United States: shifting paradigms. Clin Microbiol Rev. 1998;11(3):497-513. doi: 10.1128/ CMR.11.3.497

17. Krohn MA, Thwin SS, Rabe LK, Brown Z, Hillier SL. Vaginal colonization by Escherichia coli as a risk factor for very low birth weight delivery and other perinatal complications. J Infect Dis. 1997;175(3):606-610. doi: 10.1093/infdis/175.3.606 